\title{
Glaucoma detection of retinal images based on boundary segmentation
}

\author{
Noraina Alia Zainudin ${ }^{1}$, Ain Nazari ${ }^{2}$, Mohd Marzuki Mustafa ${ }^{3}$, Wan NurShazwani Wan Zakaria ${ }^{4}$, \\ Nor Surayahani Suriani ${ }^{5}$, Wan Nur Hafsha Wan Kairuddin ${ }^{6}$ \\ ${ }^{1}$ Faculty of Electrical and Electronic Engineering, Universiti Tun Hussein Onn Malaysia, Malaysia \\ ${ }^{2,4}$ Department of Mechatronic and Robotic Engineering, Faculty of Electrical and Electronic Engineering, Universiti Tun \\ Hussein Onn Malaysia, Malaysia \\ ${ }^{3}$ Department of Electrical, Electronic \& System Engineering, Faculty of Engineering and Built Environment, \\ Universiti Kebangsaan Malaysia, Malaysia \\ ${ }^{5}$ Department of Computer Engineering, Faculty of Electrical and Electronic Engineering, \\ Universiti Tun Hussein Onn Malaysia, Malaysia \\ ${ }^{6}$ Department of Electronic Engineering, Faculty of Electrical and Electronic Engineering, \\ Universiti Tun Hussein Onn Malaysia, Malaysia
}

\section{Article Info}

Article history:

Received Jun 20, 2019

Revised Oct 21, 2019

Accepted Nov 5, 2019

\section{Keywords:}

Adaptive Thresholding

Glaucoma

Morphological

Segmentation

\begin{abstract}
The rapid growth of technology makes it possible to implement in immediate diagnosis for patients using image processing. By using morphological processing and adaptive thresholding method for segmentation of optic disc and optic cup, various sizes of retinal fundus images captured through fundus camera from online databases can be processed. This paper explains the use of color channel separation method for pre-processing to remove noise for better optic disc and optic cup segmentation. Noise removal will improve image quality and in return help to increase segmentation standard. Then, morphological processing and adaptive thresholding method is used to extract out optic disc and optic cup from fundus image. The proposed method is tested on two publicly available online databases: RIM-ONE and DRIONS-DB. On RIM-ONE database, the average PSNR value acquired is 0.01891 and MSE is 65.62625. Meanwhile, for DRIONS-DB database, the best PSNR is 64.0928 and the MSE is 0.02647 . In conclusion, the proposed method can successfully filter out any unwanted noise in the image and are able to help clearer optic disc and optic cup segmentation to be performed.
\end{abstract}

Copyright $(2020$ Institute of Advanced Engineering and Science. All rights reserved.

\section{Corresponding Author:}

Ain Nazari,

Department of Mechatronic \& Robotic Engineering,

Faculty of Electrical and Electronic Engineering,

Universiti Tun Hussein Onn Malaysia, Johor, Malaysia

Email: ain@uthm.edu.my

\section{INTRODUCTION}

Glaucoma is one of the factors for irreversible blindness in the world. Visual field loss, optic disc cupping, and in most instances an increased intraocular pressure are the main features of glaucoma [1]. It is originated from increasing pressure called intraocular pressure (IOP). The pressure happens because of the leftover fluid that is gradually accumulated in the front part of the eye. The liquid cause increasing pressure within the eyeball and eventually lead to optic nerve damage.

If immediate treatment was not delivered, it could lead to permanent eye damage and untreatable vision loss. Figure 1 shows glaucoma progression from normal vision until extreme glaucoma. 


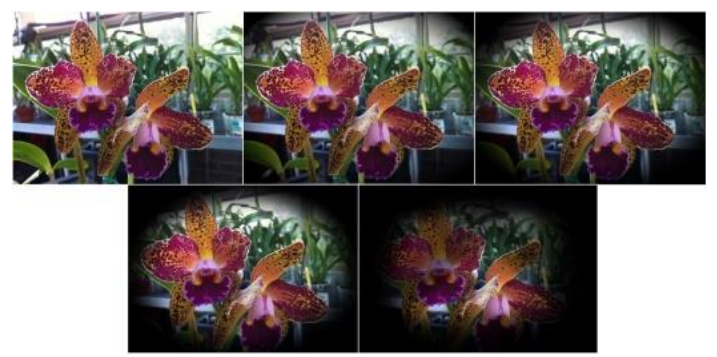

Figure 1. Normal vision progression into extreme glaucoma [2]

However, not all high eye pressure caused glaucoma. Sometimes glaucoma can also happen to normal eye pressure as well. This depends on the level of pressure employed on the particular optic nerve. This pressure was usually measured using Goldmann Applanation Tonometry. Goldmann Applanation Tonometry was appraised as the main standard for IOP measurement [3].

Generally, there are two common glaucoma types which are primary open angle glaucoma (POAG) and angle closure glaucoma (ACG). About two thirds of glaucoma cases are POAG while the rest are ACG cases [1, 4-7]. Commonly, some well-developed health facility will provide free screening to detect glaucoma in patients [8]. This screening will be conducted using a fundus camera and performed by approved professionals and medical practices.

For glaucoma infected eyes, it can be seen from the size of the optic cup and optic disc as illustrated in Figure 2. Optic disc is the path where ganglion cell axons exit and leave the eye. Optic cup is situated in front of the optic disc. It is shaped like a small crater. Conventionally, the size of optic cup is smaller than optic disc. Only in glaucoma infected eye, the optic cup size increases and grow larger. This is due to elevation of IOP. Besides, blood flow to the optic nerve is loss and nerve fibers slowly dying. The normal size for optic cup is 0.3 to 0.4 while glaucomatous eye have cup size of 0.7 to 0.9 [9].
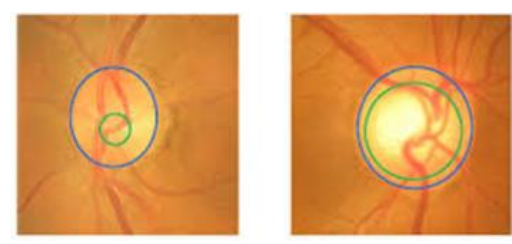

Figure 2. Normal cup size versus glaucomatous cup size [10]

The algorithm for this software that will be developed can perform the image processing techniques needed for the detection of glaucoma. There are two important steps completed. The steps are pre-processing phase and segmentation phase shown in Figure 3.

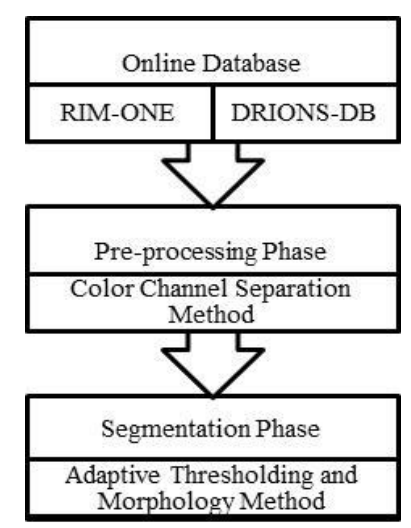

Figure 3. Block diagram of proposed system 


\section{RESEARCH METHOD}

The images used in this project were obtained from two online databases which are RIM-ONE [11] and DRIONS-DB [12]. RIM-ONE database consists of 169 Optic Nerve Head (ONH) images retrieved from 169 full fundus images of different subjects. Figure 4 displayed examples of images from RIM-ONE. Domain experts have categorized the images into five different subsets:

a. 11 images of Ocular hypertension (OHT).

b. 14 images of Deep glaucoma.

c. 118 images of Normal eye (non-glaucomatous).

d. 12 images of Early glaucoma.

e. 14 images of Moderate glaucoma.

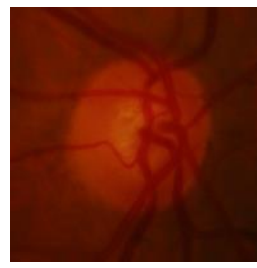

(i)

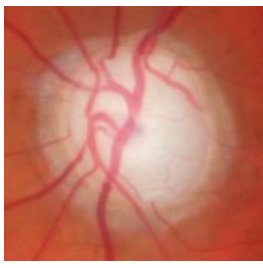

(ii)

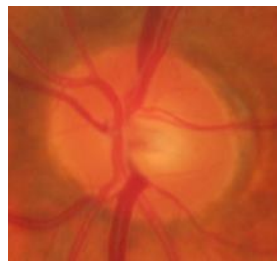

(iii)

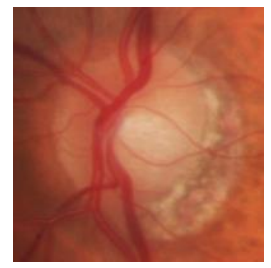

(iv)

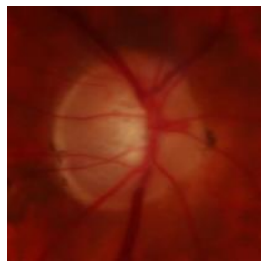

(v)

Figure 4. The examples of images from RIM-ONE database for (i) Ocular hypertension (OHT) (ii) Deep glaucoma (iii) Normal eye (non-glaucomatous) (iv) Early glaucoma and (v) Moderate glaucoma

Meanwhile th next database which is DRIONS-DB demonstrates the segmentation of the optic nerve head in retinal fundus images. It was gathered by 2 medical experts. It has 2 ground truths for each set of 110 colour digital retinal images. Figure 5 illustrate the examples of images from this database.
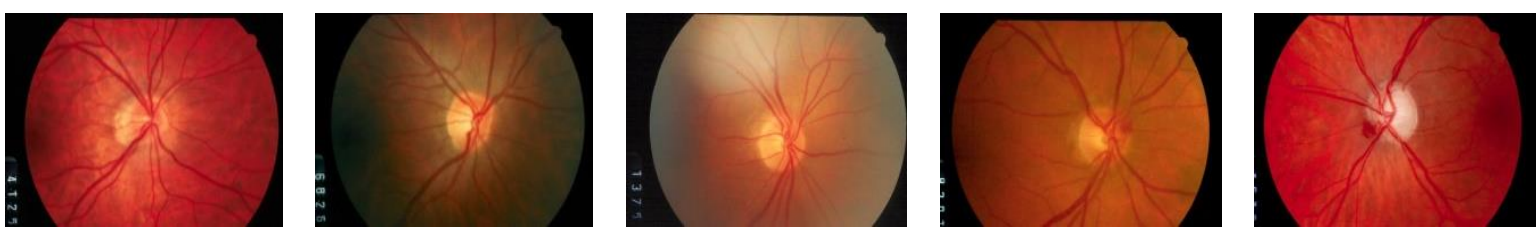

Figure 5. Samples of images from DRIONS-DB database

\subsection{Pre-Processing}

Pre-processing image is a common early method to obtain improved and enhanced image and to draw out useful information from it. Pre-processing usually involve images with the lowest level of abstraction. The aim of pre-processing is to remove noise, correct some of the degradation in image, suppresses unwanted distortions and amplifies image features important for further processing.

For pre-processing, the method used is color channel separation method [13]. The fundus images obtained from databases are split into three color channels. Normally used color channels are red, green, and blue color channels (RGB). The RGB components usually regarded as three sub-sources of single color source [14]. 24-bit RGB image can give storage pixels with conventional brightness intensities of 0 until 255 [15]. It first started with black color and the light emission increase as correspond to added colors. Figure 6 shows examples of images obtained as the outcome of color channel separation method.

One of the color channels is chosen and then processed further into grayscale. For this system, red color channel selected and undergoes the next process. 

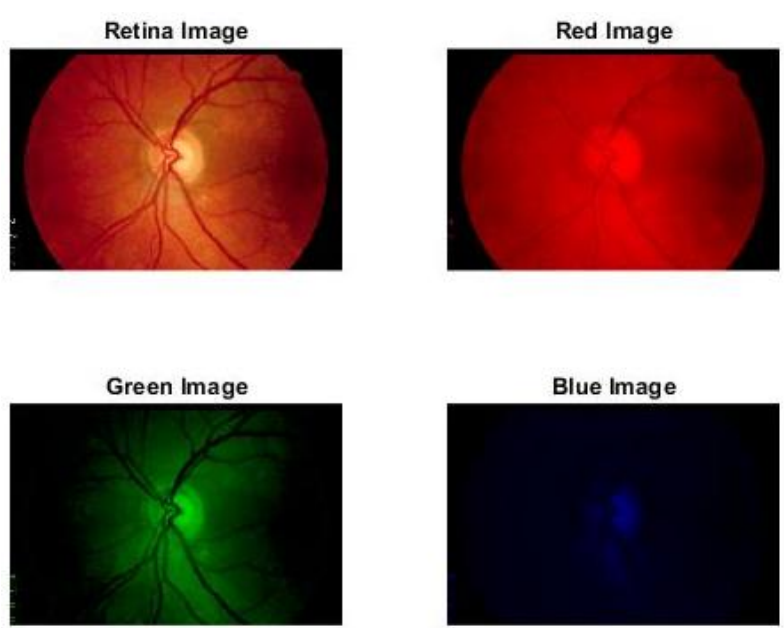

Figure 6. Fundus image after separated into three color channels

\subsection{Segmentation Phase}

The next process are segmenting and extracting important features from the grayscale image. obtained from pre-processing. Segmentation phase will separate an image into pixels with similar attributes. The pixels then accumulated into its distinguishable regions. This distinguishable regions need to have large relation to characteristic of interest for an accurate image interpretation. Typically for glaucoma, the important features that need to be extracted are blood vessels and optic disc.

In this phase, the obtained grayscale image is converted into binary disc image. Binary disc image demonstrate raw disk images after conversion from grayscale image.

After that, a further processed detailed image almost similar to binary disc image acquired using Morphological Processing method [16]. This image acquired is more refined that previous binary disc image existed as it improves and enhance image so that further processing can be made with ease. Generally, there are two formulas used in Morphological Processing method [16]. Each one represents dilation operation and erosion operation. Dilation operation is a type of operation that increase object in a binary image whether in terms of size or thickness meanwhile erosion operation is the operation that does the opposite of dilation method for example the operation reduce object's size or thickness in binary image. These two operations are very important in increasing the accuracy of Morphological Processing method. It is expressed as:

$$
\mathrm{X} \oplus \mathrm{B}=\mathrm{X}+\mathrm{b}=\{\mathrm{x}+\mathrm{b}: \mathrm{x} \in \mathrm{X} \& \mathrm{~b} \in \mathrm{B}\}
$$

Where $\mathrm{X}$ is any grayscale shape and B is symmetric structuring element.

$$
\mathrm{X} \Theta \mathrm{B}=\mathrm{X}-\mathrm{b}=\{\mathrm{z}:(\mathrm{B}+\mathrm{z}) \subseteq \mathrm{X}\}
$$

Symmetric structuring element is contained inside input set $\mathrm{X}$ as output of erosion is also translated as set of translation points. Figure 7 shows the example of dilation operation while Figure 8 represents erosion operation.

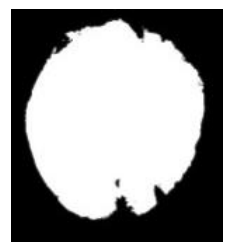

(i)

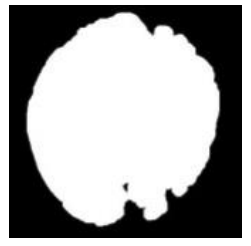

(ii)

Figure 7. Dilation operation (i) before operation (ii) after operation 


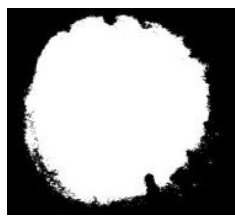

(i)

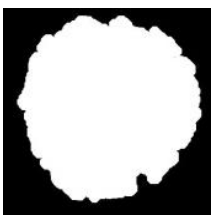

(ii)

Figure 8. Erosion operation (i) before operation (ii) after operation

Furthermore, the segmented disk image showing optic disc using Adaptive Thresholding method is secured. By using this method, optic disc can be carefully and clearly be extracted out. Optic disc is the most important characteristic in diagnosing glaucoma [17]. This owe to the fact that glaucoma is very hard to be roughly detected with only our eyes. It needs proper eye examination with suitable machine. Optic disc can show one of the obvious sign in diagnosing glaucoma.

Lastly, the segmentation was made to identify optic cup as well. Optic cup is also very important characteristics in glaucoma detection. Its size can determine whether an eye is healthy or glaucomatous. From images obtained, it is proven that the optic cup of glaucomatous eye is bigger than optic cup of normal eye [18-20]. All the images for each process can be viewed in Table 1.

Circular Hough Transform (CHT) method was commonly implemented as segmentation of optic disc [21]. By comparing to the proposed method, it can be seen that Adaptive Thresholding and Morphology method is more accurate as it also extract out optic cup and optic disc of retina fundus image. The CHT method only capable to show and illustrates countless circles as in shown in Figure 9 (i) while in Figure 9 (ii), one circle to detect optic disc was picked as maximum energy in the vicinity.

Table 1. Images Obtained after Each Process

\begin{tabular}{ccccc}
\hline \multicolumn{2}{c}{ RIM-ONE } \\
\hline $\begin{array}{c}\text { Original } \\
\text { image }\end{array}$ \\
$\begin{array}{c}\text { Grayscale } \\
\text { image }\end{array}$ \\
$\begin{array}{c}\text { Binary } \\
\text { disc image } \\
\text { disc image }\end{array}$ \\
$\begin{array}{c}\text { Binary cup } \\
\text { image }\end{array}$
\end{tabular}

the best approximate circle (maximum energy in the vicinity) [21] 


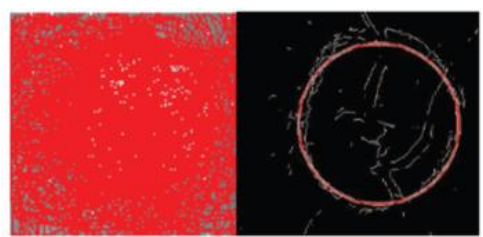

(i)

(ii)

Figure 9. (i) Demonstrates the various circles (in red) detected by the Hough Transform (ii) Shows

\subsection{Performance Metrics}

There are two parameters calculated for performance metrics for pre-processing. The Peak Signal to Noise Ratio (PSNR) and the Mean Square Error (MSE) are the two error metrics used to compare image compression quality. The calculation for PSNR and MSE is completed to compare the accuracy of the preprocessing method used [22-23].

Peak signal-to-noise ratio (PSNR) shows the ratio of power limit to the power of same image with noise. The PSNR calculate the peak signal-to-noise ratio between two images. It is normally regarded in logarithmic decibel scale (dB). However, it also can be calculated in percent (\%). MAXI (maximum possible pixel value) of the image used is 255 because the image is uint8 [24].

As shown in (3) below is the formula for calculating PSNR and (4) is the formula to calculate MSE.

$$
\begin{aligned}
& \mathrm{PSNR}=10 \times \llbracket \log \rrbracket \_10\left(\left[\mathrm{MAXI} \rrbracket^{\wedge} 2 / \mathrm{MSE}\right)\right.
\end{aligned}
$$

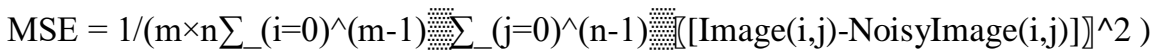

\section{RESULTS AND ANALYSIS}

It can be concluded that for RIM-ONE database, the PSNR is 0.01891 and MSE is 65.62625. Meanwhile, for DRIONS-DB database, the PSNR is 64.0928 and the MSE is 0.02647 . The results obtained clearly stated that the PSNR value obtained from after pre-processing is higher than the original image. The higher the PSNR value, the higher the level of audio signal than level of noise [25]. So, it is proven that the image quality after pre-processing is better than original image as noise was removed [26]. Table 2 indicates the PSNR value of original image before and after pre-processing was made.

Table 2. The PSNR Value Before and after Pre-Processing

\begin{tabular}{lllllll}
\hline & RIM-ONE & \multicolumn{5}{c}{ DRIONS-DB } \\
\cline { 2 - 7 } & $\begin{array}{l}\text { Image } \\
\text { sample } \\
\text { from } \\
\text { database }\end{array}$ & $\begin{array}{l}\text { MSE } \\
\text { value (\%) }\end{array}$ & $\begin{array}{l}\text { PSNR } \\
\text { value (\%) }\end{array}$ & $\begin{array}{l}\text { Image } \\
\text { sample from } \\
\text { database }\end{array}$ & $\begin{array}{l}\text { MSE } \\
\text { value (\%) }\end{array}$ & $\begin{array}{l}\text { PSNR } \\
\text { value (\%) }\end{array}$ \\
N & Im133 & 0.0158 & 66.1523 & image_001 & 0.0366 & 62.4979 \\
o & Im152 & 0.0196 & 65.2033 & image_002 & 0.0314 & 63.1555 \\
r & Im160 & 0.0197 & 65.1949 & image_003 & 0.0348 & 62.7135 \\
m & Im161 & 0.0246 & 64.2238 & image_031 & 0.0204 & 65.0375 \\
a & Im169 & 0.0278 & 63.6834 & image_062 & 0.0113 & 67.6144 \\
l & Im013 & 0.0103 & 68.0137 & image_006 & 0.0283 & 63.6138 \\
G & & & & & & \\
1 & Im016 & 0.0120 & 67.3244 & image_008 & 0.0275 & 63.7350 \\
a & Im038 & 0.0151 & 66.3510 & image_024 & 0.0239 & 64.3449 \\
u & Im103 & 0.0309 & 63.2242 & image_047 & 0.0246 & 64.2258 \\
c & Im120 & 0.0133 & 66.8915 & image_092 & 0.0259 & 63.9897 \\
o & & & & & & \\
m & & 0.01891 & 65.62625 & & 0.02647 & 64.0928 \\
a & & & & & & \\
Average (\%) & & & & & & \\
\hline
\end{tabular}

\section{CONCLUSION}

As conclusion, the segmentation and detection of glaucoma is successfully completed. The methods used for preprocessing and segmentation works perfectly and are able to give satisfactory result. For future 
work, the next process must be conducted. For instances, next process should involve calculating the size of optic cup and optic disc then proceed to classification into two common different types of glaucoma. Besides, a MATLAB GUI can also be developed in the future for a more efficient and accurate glaucoma detection system.

\section{ACKNOWLEDGEMENTS}

The author would like to thank Research, Innovation, Commercialization and Consultation Management (ORICC) Universiti Tun Hussein Onn Malaysia (H203 Tier 1 Fund) and Registrar Office for sponsoring the research and development of this project.

\section{REFERENCES}

[1] Marais, A., \& Osuch, E., "The medical management of glaucoma. South African Family Practice", May 2017, 9(2):6-13.

[2] http://hasznaltmobil.info/mmp/k/keratoconus-vision-simulator/. Retrieved April $18^{\text {th }}, 2019$.

[3] Athar Zareei, MS., Mohammad Reza Razeghinejad, MD., Mohammad Hosein Nowroozzadeh, MD., Yadollah Mehrabi, OD., \& Mohammad Aghazadeh-Amiri, OD., "Intraocular Pressure Measurement by Three Different Tonometers in Primary Congenital Glaucoma" Journal of Ophthalmic \& Vision Research, May 2015; 10(1): 43- 48.

[4] Schellack, N., Schellack, G., \& Bezuidenhout, S., "Glaucoma: a brief review”, January 2015, Vol 82 No 5.

[5] H. Fu, Y. Xu, S. Lin, X. Zhang, D. Wong, J. Liu, and A. Frangi, "Segmentation and Quantification for AngleClosure Glaucoma Assessment in Anterior Segment OCT," IEEE Trans. Med. Imag., vol. 36, no. 9, pp. 1930-1938, 2017.

[6] Abu-Amero K., Kondkar A.A., Chalam K.V. "An updated review on the genetics of primary open angle glaucoma“. Int J Mol Sci. 2015; 16(12): 28886-28911.

[7] https://www.researchgate.net/figure/a-Open-angle-glaucoma-b-angle-closure-glaucoma_fig1_323248057. Retrieved April 18th, 2019.

[8] Hameed, S. A., Habaebi, M. H., Haddad, A., "Application of Mobile Cloud Computing in Emergency Health Care”, Bulletin of Electrical Engineering and Informatics (BEEI), Vol 8, No 3, September 2019.

[9] Bhardwaj, K., (2015, October 26). Retrieved February 26, 2019 from Slideshare.net for Primary open angle glaucoma: https://www.slideshare.net/smithwill1985/primary-open-angle-glaucoma-54398174.

[10] https://goo.gl/images/z54xAz. Retrieved February 26th, 2019.

[11] Saavedra, J. F., Miranda, S. A., de la Rosa, J. L., Batista, F. J., Regalado, O. N., \& González, S. d. (n.d.). "RIM-ONE Database". Retrieved September 24, 2018, from Medical Image Analysis Group: http://medimrg.webs.ull.es/.

[12] Feijoo, J. G., de la Casa, J. M., Servet, H. M., Zamorano, M. R., Mayoral, M. B., \& Suárez, E. J. (2009, August 21). Retrieved August 19, 2018, from DRIONS-DB: Digital Retinal Images for Optic Nerve Segmentation Database: http://www.ia.uned.es/ ejcarmona/DRIONS-DB.html.

[13] Nazari, A., Mustafa, M. M., \& Zulkifley, M. A., "Segmentation of Retinal Blood Vessels by Top-Hat Multi Scale Detection for Optic Disc Removal', Jurnal Teknologi, 77(6):47-53.

[14] Kayabol, K., Kuruoglu, E. E., \& Sankur, B., "Image Source Separation using Color Channel Dependencies", In Independent Component Analysis and Signal Separation, pages 499-506, Springer, 2009.

[15] https://en.wikipedia.org/wiki/Channel_(digital_image). Retrieved February 21st, 2019.

[16] Tambe1, S. B., Kulhare, D., Nirmal, M. D., \& Prajapati, G., "Image Processing (IP) Through Erosion and Dilation Methods", International Journal of Emerging Technology and Advanced Engineering, Volume 3, Issue 7, July 2013

[17] Wisaeng, K., Hiransakolwong, N., \& Pothiruk, E., “Automatic Detection of Optic Disc in Digital Retinal Images”, International Journal of Computer Applications (0975-8887), Volume 90-No 5, March 2014.

[18] Arkachari, P., \& Reshma, M., "Hybrid Approach of Detection of Glaucoma with Optic Disc and Optic Cup Segmentation in Retinal Fundus Images", International Journal for Innovative Research in Science \& Technology, Volume 2, Issue 12,May 2016.

[19] https://www.haasvisioncenter.com/glaucoma. Retrieved April 18th, 2019.

[20] Gaurav Kumar, Pradeep Kumar Bhatia, "A Detailed Review of Feature Extraction in Image Processing System," International Conference on Advanced Computing \& Communication Technologies, 2014.

[21] Gopalakrishnan, A., Almazroa, A., Raahemifar, K., \& Lakshminarayanan, V., "Optic Disc Segmentation using Circular Hough Transform and Curve Fitting", November 2015.

[22] Raushan Kumar, R., Sharma, G., \& Sanduja, V., "A Real Time Approach to Compare PSNR and MSE Value of Different Original Images and Noise ( Salt and Pepper, Speckle, Gaussian) Added Images”, International Journal of Latest Technology in Engineering, Management \& Applied Science (IJLTEMAS), Volume VII, Issue I, January 2018.

[23] Batra, B., Singh, S., Sharma, J, \& Arora, S. M., "Computational analysis of edge detection operators", International Journal of Applied Research, 2016; 2(11): 257-262.

[24] Pinki \& Mehra, R.," Estimation of the Image Quality under Different Distortions", International Journal Of Engineering And Computer Science, ISSN: 2319-7242, Volume 5 Issues 7 July 2016, Page No. 17291-17296. 
[25] Jeyavathana1, R. B., Balasubramanian, R., \& Pandian, A. A., "A Survey: Analysis on Pre-processing and Segmentation Techniques for Medical Images", International Journal of Research and Scientific Innovation (IJRSI), Volume III, Issue VI, June 2016, ISSN 2321-2705.

[26] Amedo, A. O., Koomson, N. Y., Acquah, E. K., Pascal, T. M., Atuahene, J., Akowuah, P. K., Djeagbo, P. T., \& Baafi, R., "Comparison of the clinical estimation of cup-to-disk ratio by direct ophthalmoscopy and optical coherence tomography", Therapeutic Advances in Ophthalmology, January 2019.

\section{BIOGRAPHIES OF AUTHORS}
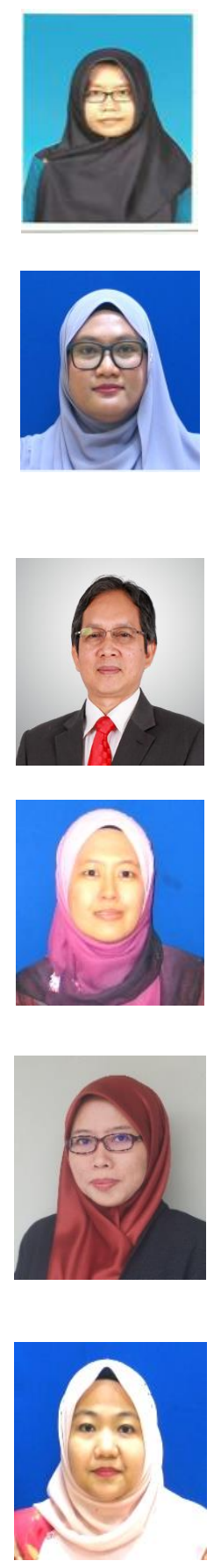

Noraina Alia Zainudin was born on 31st July 1995 at Hospital Kuala Lipis, Pahang. She is currently pursuing her Bachelor Degree in Electronic Engineering at Universiti Tun Hussein Onn Malaysia (UTHM). Previously, she graduated from Kolej Matrikulasi Perak (KMPk) in Science. Her current and previous research interests are related to medical research. Currently, she is an active member of The Institutes of Engineers Malaysia (IEM).

Ain Nazari received Diploma and B. Eng degree in Electrical Engineering in 2005 and 2008 from Universiti Tun Hussein Onn Malaysia (UTHM), M. Eng (Mechatronics and Automatic Control) and $\mathrm{PhD}$ in Electronics and Computer System in 2010 and 2018 from Universiti Teknologi Malaysia (UTM) and Universiti Kebangsaan Malaysia (UKM), respectively. In 2008 she joined UTHM and is currently a lecturer in the Faculty of Electrical and Electronic Engineering. Her main research interests are in image processing specialize in medical image and computer vision.

Mohd Marzuki Mustafa received the B.Eng. degree in Electrical Engineering in 1985, and the M.Sc. and PhD. degrees in Control Systems Engineering in 1986 and 1989 from University of Manchester Institute of Science and Technology, United Kingdom, and University of Salford, United Kingdom, respectively. In 1984, he joined the Universiti Kebangsaan Malaysia and is currently a professor in the Faculty of Engineering and Built Environment. His main research interests are in computer control systems \& Instrumentation and Machine Vision.

Wan NurShazwani Wan Zakaria received B.Eng (2007) in Electronics and Mechanical Engineering from Chiba University and MSc in Mechatronics (2008) and PhD in Mechanical and System Engineering (2012) from Newcastle University. She is currently a lecturer in Faculty of Electrical and Electronic Engineering, Universiti Tun Hussein Onn Malaysia. Her current interests include Medical Robotics System specifically on development of robot force control, Image Processing and Computer Aided Diagnosis, and development of Wearable Device. She is author and co-author of several journal papers and conference proceedings.

Nor Surayahani Suriani is Senior Lecturer in Universiti Tun Hussein Onn Malaysia (UTHM). She received her $\mathrm{PhD}$ in Electronics and Computer System from Universiti Kebangsaan Malaysia (UKM) in 2015, and finished her Master (Universiti Teknologi Malaysia) and Bach. Eng. (Universiti Putra Malaysia) in Electronics and Communication in 2007 and 2003, respectively. Her research interest focuses on computer vision, deep learning, image processing and bioinspired visual cortex algorithm development. She has published in Q1 impact factor and Scopus journals mainly in image processing and computer vision areas. She also a member in national Board of Engineers.

Wan Nur Hafsha Wan Kairuddin received her M.Eng (Electrical Engineering) from Universiti Tun Hussein Onn Malaysia (UTHM) in 2016, and B.Eng (Electronics Engineering) in 2005 from Universiti Sains Malaysia (USM). She is currently an Instructor Engineer in Universiti Tun Hussein Onn Malaysia (UTHM). Her research interest focuses on medical image processing and healthcare system development. She received certification as Professional Technologist in 2019 from Malaysia Board of Technologies. She also a member of national Board of Engineers. 